\title{
Cardiac troponin T mutations promote life-threatening arrhythmias
}

\author{
Céline Fiset ${ }^{1}$ and Wayne R. Giles ${ }^{2}$ \\ ${ }^{1}$ Research Center, Montreal Heart Institute, Faculty of Pharmacy, University de Montréal, Montreal, Quebec, Canada. \\ ${ }^{2}$ Faculty of Kinesiology, University of Calgary, Calgary, Alberta, Canada.
}

\begin{abstract}
Mutations in contractile proteins in heart muscle can cause anatomical changes that result in cardiac arrhythmias and sudden cardiac death. However, a conundrum has existed because mutations in one such contractile protein, a so-called $\mathrm{Ca}^{2+}$ sensor troponin $\mathrm{T}(\mathrm{TnT})$, can promote ventricular rhythm disturbances even in the absence of hypertrophy or fibrosis. Thus, these mutations must enhance abnormal electrophysiological events via alternative means. In this issue of the JCI, Baudenbacher et al. report a novel mechanism to explain this puzzle (see the related article, doi:10.1172/ JCI36642). They show that a selected TnT mutation in the adult mouse heart can markedly increase the sensitivity of cardiac muscle myofilaments to $\mathrm{Ca}^{2+}$ and enhance the susceptibility to arrhythmia, even in the absence of anatomical deformities. As these same mutations can cause some forms of arrhythmias in humans, these findings are of both basic and translational significance.
\end{abstract}

Cardiomyopathy is defined as a heterogeneous group of diseases of the myocardium that are associated with mechanical and/or electrical dysfunction $(1,2)$. Ventricular hypertrophy and fibrosis and/or inappropriate dilation in the absence of coronary disease, hypertension, valvular disease, or congenital heart disorders are commonly observed. In mammalian hearts, cardiomyopathies may be either inherited or acquired and often result in or are accompanied by disturbances in ventricular rhythms. The significant incidence of cardiomyopathy in the older and diabetic populations has lead to intensive research into the cellular and molecular causes of this syndrome.

These findings, however, have raised a fundamental question related to whether anatomical changes are a prerequisite or a consequence of the life-threatening rhythm disturbances observed in association with cardiomyopathies. One approach to solving this problem is based on application of genetic techniques that alter either the amount or the functional properties of the contractile proteins within the cardiac cell (myocyte). In particular, investigators have shown that alteration (mutation) of individual amino acids in the contractile

Nonstandard abbreviations used: $\operatorname{Tn} \mathrm{T}$, troponin $\mathrm{T}$.

Conflict of interest: The authors have declared that no conflict of interest exists.

Citation for this article: J. Clin. Invest. doi:10.1172/ JCI37787. protein that is the major $\mathrm{Ca}^{2+}$ sensor, troponin $\mathrm{T}(\mathrm{Tn} T)$, can lead to life-threatening rhythm disturbances in the absence of any detectable changes in myocyte structure or increase in fibroblasts within the heart $(3,4)$. Interestingly, however, this mutation results in the contractile elements responding to changes in intracellular $\mathrm{Ca}^{2+}$ in a way that reflects a sensitization or overall increased sensitivity to changes in intracellular $\mathrm{Ca}^{2+}$. This change is of fundamental significance to the heart, since, on a beat-by-beat basis, $\mathrm{Ca}^{2+}$ enters the cell through $\mathrm{Ca}^{2+}$ channels in the surface membrane, and this $\mathrm{Ca}^{2+}$ entry promotes rapid and large release of $\mathrm{Ca}^{2+}$ from the main intracellular store, the sarcoplasmic reticulum. This rapid increase in intracellular $\mathrm{Ca}^{2+}$ is detected by the $\mathrm{Ca}^{2+}$-sensitive protein TnT, which then undergoes a structural alteration to allow the contractile filaments actin and myosin to interact. This produces a cellular contraction and the heart beat.

A multidisciplinary study by Baudenbacher, Knollmann, and colleagues (3) in this issue of the JCI advances our present understanding of the pathophysiological basis for cardiomyopathy in the absence of cardiac anatomical abnormalities such as hypertrophy or fibrosis. In this study and previous work from this laboratory (4), a combination of pharmacologic and transgenic approaches, both in vitro and in vivo, were used to identify and characterize the electrophysiologic sequelae (e.g., ventricular tachycardia) associated with cardiomyopathy. Targeted mutations of individual amino acids within the main $\mathrm{Ca}^{2+}$ sensor, $\operatorname{TnT}$, and in selected regions of sarcomere thin filament proteins are well known to result in alterations in their $\mathrm{Ca}^{2+}$ sensitivity $(5,6)$. It is noteworthy that selective changes of this kind can in fact result in either increased or decreased $\mathrm{Ca}^{2+}$ sensitivity (7), as well as significant changes in myocyte or tissue metabolism/energetics (8). The mutation that is the focus of the Baudenbacher et al. study (3) results in significantly increased $\mathrm{Ca}^{2+}$ sensitivity of the contractile myofilaments in the mouse heart. This increase in $\mathrm{Ca}^{2+}$ sensitivity, by as yet undefined mechanism(s), results in altered patterns of ventricular electrical activity. Specifically, there are changes in conduction velocity and the excitability of the ventricle, as demonstrated by its ability to react to applied stimuli (shortened effective refractory period). These changes combine to result in a markedly increased tendency for inducible tachyarrhythmias (clusters of abnormally rapid beating), following programmed stimulation or the application of the cardiac adrenergic stimulant isoproterenol. The authors also observed marked changes in cellular electrophysiology; the action potential shortened dramatically. All of these electrophysiological changes were reported to be closely mimicked by application of EMD 50733, a pharmacological agent that is considered to be a selective myofilament $\mathrm{Ca}^{2+}$ sensitizer (9). In addition, the incidence of ventricular rhythm disturbances was markedly reduced or eliminated by administration of the $\mathrm{Ca}^{2+}$ desensitizing compound blebbistatin, which prevents actin-myosin interactions $(10,11)$.

\section{Defining the link between $\mathrm{Ca}^{2+}$ sensitivity and} ventricular electrophysiology

Selected mutations in $\mathrm{TnT}$ in the mouse ventricular myocyte can result in a cardiomyopathic syndrome, including hypertro- 
phy and fibrosis as well as an increased tendency for ventricular rhythm disturbances and $\mathrm{Ca}^{2+}$-dependent changes in action potential morphology (4). Baudenbacher et al. (3) now demonstrate that the increased susceptibility to arrhythmia can be observed in the absence of any detectable cardiac hypertrophy or fibrosis. They report that the increased risk of ventricular tachycardia is directly proportional to the enhancement of $\mathrm{Ca}^{2+}$ sensitization caused by the selected TnT mutation.

It is very important, therefore, to consider in some detail the $\mathrm{Ca}^{2+}$ sensitivity findings reported in the current study (3). The authors report a significantly enhanced steady-state $\mathrm{Ca}^{2+}$ sensitivity based on force measurements from chemically skinned ventricular trabeculae from adult mouse ventricles. One would expect that this change may alter overall $\mathrm{Ca}^{2+}$ buffering or homeostasis within each myocyte (12). As a consequence important changes in intracellular $\mathrm{Ca}^{2+}$ could take place during both the contractile (systolic) and the relaxation (diastolic) phases of ventricular function. Such changes in intracellular $\mathrm{Ca}^{2+}$ levels are known to have significant electrophysiological effects.

If $\mathrm{Ca}^{2+}$ levels were to increase, it would be expected that the predominant $\mathrm{Ca}^{2+}$ current in the mouse ventricle may decrease in size and turn off, or inactivate, more quickly (12). This would be consistent with the action potential data reported by Baudenbacher et al. (3), which demonstrated action potential shortening: a loss of the plateau and marked shortening of duration during early repolarization. Changes in intracellular $\mathrm{Ca}^{2+}$ concentration would also alter the transport of $\mathrm{Ca}^{2+}$ out of the cell by the so-called $\mathrm{Na}^{+} / \mathrm{Ca}^{2+}$ exchange mechanism (12), and this electrogenic transporter can alter ventricular excitability.

Experimental approaches applied in rat and mouse myocytes, in which genetic manipulations or pharmacological treatment have caused changes in contractile filament $\mathrm{Ca}^{2+}$ sensitivity, can also cause altered myocyte metabolism/energetics (8, 13). If these metabolic changes resulted in significant decreases in either the spatially averaged or localized levels of ATP, it would be reasonable to expect activation of ATPsensitive $\mathrm{K}^{+}$current and alteration of both the $\mathrm{Na}^{+} / \mathrm{Ca}^{2+}$ exchanger and the $\mathrm{Na}^{+} / \mathrm{K}^{+}$ pump. Previous work has demonstrated that the molecular transcripts responsible for generating ATP-sensitive $\mathrm{K}^{+}$currents are expressed in the adult mouse ventricle and that either pharmacological or genetic alteration in this current can significantly alter the ventricular electrophysiological characteristics. These alterations can be either protective or proarrhythmic $(14,15)$.

\section{Action potential waveform modulates intracellular $\mathbf{C a}^{2+}$ levels}

Our groups (16) and others have shown that action potential shape changes similar to those described by Baudenbacher et al. (3) can dramatically alter the associated intracellular $\mathrm{Ca}^{2+}$ transient that triggers each cardiac contraction. Specifically, shortening the action potential decreases the $\mathrm{Ca}^{2+}$ influx carried by L-type $\mathrm{Ca}^{2+}$ channels. Since this $\mathrm{Ca}^{2+}$ influx is the "trigger" for $\mathrm{Ca}^{2+}$ release from the sarcoplasmic reticulum, the intracellular $\mathrm{Ca}^{2+}$ transient is decreased substantially by action potential shortening in the mammalian heart $(17,18)$. It is also known that this pattern of events differs significantly between myocytes from the right versus the left ventricle in the adult mouse heart (19). The decreased action potential duration reported by Baudenbacher et al. (3) would be predicted to result in a significant decrease in contractile strength or negative inotropic effect. This would be consistent with one of the principle features of pronounced cardiomyopathy.

The overall $\mathrm{Ca}^{2+}$ homeostasis (or buffering capacity in the adult ventricular myocyte) is modulated strongly by the myocyte resting membrane potential $(20,21)$. This is a consequence of the link between membrane potential and activity (voltage dependence) of $\mathrm{Na}^{+} / \mathrm{Ca}^{2+}$ exchange under physiological conditions. These fundamental electrophysiological principles and the recent report (22) of a strong link between membrane potential, $\mathrm{Na}^{+} / \mathrm{Ca}^{2+}$ exchange, and associated changes in action potential morphology and incidence of rhythm disturbances (in rabbit ventricle) are noteworthy. In fact, in a previous paper from the Knollmann group (4), changes in the inwardly rectifying background $\mathrm{K}^{+}$current, $\mathrm{I}_{\mathrm{K} 1}$, which generates the mouse ventricular myocyte resting potential, were reported in myocytes from animals in which TnT mutations had been made. Neither the voltage sensitive dye methodology, nor the monophasic action potential records used by Baudenbacher et al. (3) are capable of detecting small (e.g., $10 \mathrm{mV}$ ) but significant changes in resting potential. It is also noteworthy that two of the major findings in the current study depend on the compounds EMD 57033 and blebbistatin being very selective, that is, without effect on any of the time- and voltage-dependent currents or background currents in the mouse ventricle.

\section{Computational biology to the rescue}

The elegant, but also very complex, pattern of results reported by the Knollmann group $(3,4)$ need to be placed into both physiological and pathophysiological contexts. Systems biology approaches may be able to be used as an adjunct to address these integrative challenges. In fact, a comprehensive mathematical model for the action potential of the mouse ventricular myocyte has been developed by Bondarenko et al. (23). That is, systems of equations have been developed for each of the major ionic currents that underlie the mouse ventricle action potential, and these have been combined to yield useful mathematical simulations of the action potential and associated intracellular $\mathrm{Ca}^{2+}$ release and buffering.

This approach has been extended, e.g., by the Physiome Project, to provide computational platforms that are scalable (address problems at both subcellular and tissue levels) while also providing a framework for detailed considerations of the action potential, intracellular $\mathrm{Ca}^{2+}$ homeostasis, and excitation-contraction coupling (24). In silico representations of myocyte mechanical activity and myocardium metabolism, including detailed considerations of mitochondrial biochemistry and energetics, and possible linkages to myocyte $\mathrm{Ca}^{2+}$ homeostasis have also been developed $(25,26)$. These theoretical approaches may be helpful in integrating the main findings of the current study (3) with the extensive and diverse literature that must be accounted for.

At present, however, the challenge of defining the cellular mechanisms that underlie ventricular cardiomyopathy has been advanced by the work of Baudenbacher et al. (3), who have successfully separated primary changes in intracellular $\mathrm{Ca}^{2+}$ sensor mechanisms from the anatomical changes that develop later in the cardiomyopathic syndrome. These authors have also convincingly demonstrated the utility of a multidisciplinary approach to a major problem in contemporary molecular cardiology.

\section{Acknowledgments}

Experimental and theoretical work in the authors' laboratories is supported by the Canadian Institutes of Health Research and the Heart and Stroke Foundation of Canada. W.R. Giles holds a Medical Scientist 
Award from the Alberta Heritage Foundation for Medical Research. C. Fiset is a Research Scholar of the Fonds de la Recherche en Santé du Quebéc (FRSQ).

Address correspondence to: Wayne R. Giles, Faculty of Kinesiology, University of Calgary, 2500 University Drive NW, Calgary, Canada, T2N 1N4. Phone: (403) 220-5607; Fax: (403) 220-0448; E-mail:wgiles@ucalgary.ca.

1. Elliot, P., et al. 2008. Classification of the cardiomyopathies: a position statement from the European Society of Cardiology working group on myocardial and pericardial diseases. Eur. Heart J. 29:270-276.

2. Thiene, G., Corrado, D., and Basso, C. 2008. Revisiting definition and classification of cardiomyopathies in the era of molecular medicine. Eur. Heart J. 29:144-146.

3. Baudenbacher, F., et al. 2008. Myofilament $\mathrm{Ca}^{2+}$ sensitization causes susceptibility to cardiac arrhythmia in mice. J. Clin. Invest. 118:3893-3903.

4. Knollmann, B.C., et al. 2003. Familial hypertrophic cardiomyopathy-linked mutant troponin $\mathrm{T}$ causes stress-induced ventricular tachycardia and $\mathrm{Ca}^{2+}$ dependent action potential remodeling. Circ. Res. 92:428-436

5. Harada, K., and Potter, J.D. 2004. Familial hypertrophic cardiomyopathy mutations from different functional regions of troponin $T$ result in different effects on the $\mathrm{pH}$ and $\mathrm{Ca}^{2+}$ sensitivity of cardiac muscle contraction. J. Biol. Chem. 279:14488-14495.

6. Belus, A., et al. 2008. The familial hypertrophic cardiomyopathy-associated myosin mutation R403Q accelerates tension generation and relaxation of human cardiac myofibrils. J. Physiol. 586:3639-3644.

7. Robinson, P., Griffiths, P.J., Watkins, H., and Redwood, C.S. 2007. Dilated and hypertrophic cardiomyopathy mutations in troponin and alpha-tropo- myosin have opposing effects on the calcium affinity of cardiac thin filaments. Circ. Res. 101:1266-1273.

8. Ashrafian, H., Redwood, C., Blair, E., and Watkins, H. 2003. Hypertrophic cardiomyopathy: A paradigm for myocardial energy depletion. Trends Genet. 19:263-268.

9. White, J., Lee, J.A., Shah, N., and Orchard, C.H. 1993. Differential effects of the optical isomers of EMD 53998 on contraction and cytoplasmic $\mathrm{Ca}^{2+}$ in isolated ferret cardiac muscle. Circ. Res. 73:61-70.

10. Dou, Y., Arlock, P., and Arner, A. 2007. Blebbistatin specifically inhibits actin-myosin interaction in mouse cardiac muscle. Am. J. Physiol. Cell Physiol. 93:C1148-C1153

11. Fedorov, V.V., et al. 2007. Application of blebbistatin as an excitation-contraction uncoupler for electrophysiologic study of rat and rabbit hearts. Heart Rhythm. 4:619-626.

12. Bers, D.M. 2008. Calcium cycling and signalling in cardiac myocytes. Annu. Rev. Physiol. 70:23-49.

13. Crilley, J.G., et al. 2003. Hypertrophic cardiomyopathy due to sarcomeric gene mutations is characterized by impaired energy metabolism irrespective of the degree of hypertrophy. J. Am. Coll. Cardiol. 41:1776-1782.

14. Suzuki, M., et al. 2002. Role of sarcolemmal K(ATP) channels in cardioprotection against ischemia/reperfusion injury in mice. J. Clin. Invest. 109:509-516.

15. Sasaki, N., et al. 2003. MCC-134, a single pharmacophore, opens surface ATP-sensitive potassium channels, blocks mitochondrial ATP-sensitive potassium channels, and suppresses preconditioning. Circulation. 107:1183-1188.

16. Brouillette, J., Clark, R.B., Giles, W.R., and Fiset, C. 2004. Functional properties of $\mathrm{K}^{+}$currents in adult mouse ventricular myocytes. J. Physiol. 559:777-798.

17. Bouchard, R.A., Clark, R.B., and Giles, W.R. 1995. Effects of action potential duration on excitationcontraction coupling in rat ventricular myocytes. Action potential voltage-clamp measurements. Circ. Res. 76:790-801.

18. Clark, R.B., Bouchard, R.A., and Giles, W.R. 1996.
Action potential duration modulates calcium influx $\mathrm{Na}^{+}-\mathrm{Ca}^{2+}$ exchange, and intracellular calcium release in rat ventricular myocytes. Ann. N. Y. Acad. Sci. 779:417-429.

19. Kondo, R.P., et al. 2006. Comparison of contraction and calcium handling between right and left ventricular myocytes from adult mouse heart: A role for repolarization waveform. J. Physiol. 571:131-146.

20. Bouchard, R., Clark, R.B., Juhasz, A.E., and Giles, W.R. 2004. Changes in extracellular $\mathrm{K}^{+}$concentration modulate contractility of rat and rabbit cardiac myocytes via the inward rectifier $\mathrm{K}^{+}$current

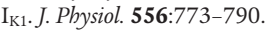

21. Pogwizd, S.M., Schlotthauer, K., Li, L., Yuan, W., and Bers, D.M. 2001. Arrhythmogenesis and contractile dysfunction in heart failure: Roles of sodium-calcium exchange, inward rectifier potassium current, and residual beta-adrenergic responsiveness. Circ. Res. 88:1159-1167.

22. Fujiwara, K., Tanaka, H., Mani, H., Takuo, N., and Takamatsu, T. 2008. Burst emergence of intracellular $\mathrm{Ca}^{2+}$ waves evokes arrhythmogenic oscillatory depolarization via the $\mathrm{Na}^{+-} \mathrm{Ca}^{2+}$ exchanger. Simultaneous confocal recording of membrane potential and intracellular $\mathrm{Ca}^{2+}$ in the heart. Circ. Res. 103:509-518.

23. Bondarenko, F.E., Szigeti, G.P., Bett, G.C., Kim, S.J., and Rasmusson, R.L. 2004. Computer model of action potential of mouse ventricular myocytes. Am. J. Physiol. Heart Circ. Physiol. 287:H1378-H1403.

24. Terkildsen, J.R., Niederer, S., Crampin, E.J., Hunter, P., and Smith, N.P. 2007. Using Physiome standards to couple cellular functions for rat cardiac excitation-contraction. Exp. Physiol. 93:919-929.

25. Niederer, S.A., and Smith, N.P. 2008. An improved numerical method for strong coupling of excitation and contraction models in the heart. Prog. Biophys. Mol. Biol. 96:90-111.

26. Cortassa, S., et al. 2006. A computational model integrating electrophysiology, contraction, and mitochondrial bioenergetics in the ventricular myocyte. Biophys. J. 91:1564-1589. 